\title{
Pegylated Liposomal Belotecan
}

National Cancer Institute

\section{Source}

National Cancer Institute. Pegylated Liposomal Belotecan. NCI Thesaurus. Code C61437.

A sterically stabilized, pegylated liposomal formulation containing belotecan, a semisynthetic analogue of campthotecin with potential antitumor activity. Belotecan inhibits the action of topoisomerase I, an enzyme that produces reversible single-strand breaks in DNA during DNA replication. This agent stabilizes the topoisomerase I and DNA complex, resulting in the inhibition of religation of DNA breaks, inhibition of DNA replication, and apoptotic cell death. The polyethylene glycol coating allows for greater plasma circulation time, thus enhancing the concentration of belotecan at the tumor site. Encapsulation of belotecan preserves the active lactone form, resulting in an increased cytotoxic effect of belotecan. 